Encuentro No. 100, 47-59, 2014

\title{
Panamá, ¿qué comemos?: reflexiones sobre soberanía alimentaria y cultura ${ }^{1}$
}

\author{
Jorge Sarsaneda del Cid*
}

Se trata de hacer un repaso de la situación de desnutrición de los pueblos indígenas en Panamá y su increíble contraste con la riqueza de nutrición que tienen en sus alimentos tradicionales. Se buscan las causas de esta situación y se plantea el debate entre seguridad y soberanía alimentaria como marco para la búsqueda de las posibles soluciones. Se parte del conocimiento de los indígenas de la zona ngäbe (oeste de Panamá) principalmente y se mencionan muchas de las capacidades nutricionales de sus alimentos, algunos incluso milenarios.

Palabras clave: indígenas / alimentación / identidad / soberanía / cultura

\section{Introducción ${ }^{2}$}

"A mi me criaron comiendo patas de rana", me comentó hace años un ngäbe, por cierto bastante robusto y fuerte. Esta expresión siempre me ha llamado la atención porque normalmente uno asocia las "ancas de rana" con platos exquisitos de restaurantes parisinos, no con la empobrecida comarca ngäbe-buglé3.

Director de Acción Cultural Ngäbe, Fe y Alegría-Panamá, Servicio Jesuita a Refugiados-Panamá y Secretario de Coordinadora Nacional de Pastoral Indígena de Panamá. Correo electrónico: chigontodobu@gmail.com

1 Este artículo fue originalmente publicado en la revista Anuario de Estudios Centroamericanos, de la Universidad de Costa Rica, en su volumen número 40 del año 2014. Agradecemos al autor, Jorge Sarsaneda, y al editor de la revista, Carlos Sandoval, por su autorización para incluirlo en esta publicación. El artículo ha sido editado para adecuarlo al formato de Encuentro.

2 El tema que se aborda es muy amplio y, por supuesto, no ha sido cubierto del todo, apenas es un primer acercamiento. Agradezco a los hermanos ngäbe y no ngäbe por sus aportes, críticas y sugerencias. Especialmente gracias a Ima Koingobu, Ome Kwradubu, Kevin Sánchez, Milton Machuca y Helena Saracho.

3 Se conoce como Comarca Ngäbe-Buglé a un territorio de casi siete mil km² -delimitado en 1997en donde habita cerca del $55 \%$ de la población indígena del pueblo ngäbe y alrededor del $35 \%$ del pueblo buglé, ambos de Panamá (Ver: Sarsaneda del Cid, 1979). 
Según los datos oficiales, el 96,3 \% de la población indígena de Panamá vive en pobreza (el 84,8 \% en pobreza extrema) y un $62 \%$ de los niños presenta un grado de desnutrición crónica. La mortalidad materna panameña entre indígenas es de las peores en América Latina (62,3 de muertes por cada mil nacidos vivos) y el índice de desarrollo humano fue de 0,447, cuando el global del país es de 0,7804 (Valdivia, 2013, pp. 415-417 y PNUD, 2010, pp. 36 y 41).

Esto resulta sencillamente escandaloso y clama por una solución pronta, pues aunque son estadísticas que se intentan "maquillar" de muchas formas, resulta imposible ocultar la realidad... a quien la quiera ver, especialmente porque son datos contradictorios y encubridores de muchas situaciones. Ante esto, en este escrito se pretende abordar no solo la situación alimentaria actual del pueblo ngäbe sino que además se buscan pautas, costumbres y raíces culturales que expliquen la permanencia de este pueblo a lo largo de los siglos, cómo se ha llegado a la situación actual y cuál es el camino para buscar soluciones permanentes.

\section{Situación inhumana durante siglos}

En el territorio panameño vive parte ${ }^{5}$ de la población de siete pueblos originarios de América Latina: ngäbe, guna, buglé, embera, wounaan, naso y bribri (ver mapa en anexo), los cuales conforman el $12 \%$ de la población total del territorio panameño ${ }^{6}$. A pesar de los siglos de dominación, rechazo y discriminación, estos pueblos conservan -en diversos grados- muchas de sus costumbres, modos de vida, ritos, creencias, idiomas, arte y vestidos; en otras palabras, muchos elementos de su identidad.

Sin embargo, hay datos que indican la situación inhumana que viven y han vivido, en todos los sentidos, durante siglos, pues no solo hubo invasión para conquistar y supuestamente "evangelizar", sino que también se impuso una cultura que, además de su idioma, escritura y religión, introyectó otras prácticas culturales alimenticias. Desde los inicios de la conquista (principios del siglo XVI) existen señales de esta imposición cultural en la zona del Darién (Mena García, 2003).

Más adelante, a inicios del siglo XVII, una carta nos relata esta situación en las palabras del cacique guaymí ${ }^{7}$ Sebastián de Silvera:

y digo que aviendo venido los españoles a poblar en estas tierras an sacado a los naturales mucha cantidad de indios unos por fuerza y otros de grado-con engaño diciendo que los enseñarían la ley de Dios y los harian christianos... y de esta manera estan todos los naturales que ay en toda esta tierra y se mueren por esos montes como animales sin saber si salvaran o no de lo

4 Haití, que es el país con el peor índice de América, tiene 0,456. Incluso, dentro de la Comarca ngäbe-buglé, hay distritos como Besigä con índice de 0,348, a nivel de los que peor están en África (Mali y Burkina Faso).

5 Parte de los pueblos guna, embera y wounaan vive en territorio de Colombia, incluso los embera han llegado al actual Ecuador. Parte de los pueblos ngäbe, bugle, naso y bribri vive en territorio de Costa Rica.

6 Según el Censo de población del 2010, la población panameña es de 3,5 millones de personas.

7 Al pueblo ngäbe antes se le llamaba "guaymí" que es una forma españolizada de decir "ngwami" que es como les decían los buglé a los ngäbe (ver: Cooke, 1982). 
qual y por el mal tratamto de los españoles se huyen y van a los montes y se vuelven a sus ydolatrias porque ven que ni los dexan vivir como es Razon sino tratándolos mal y sirviéndose de todos nosotros como si fueramos sus esclavos nos tienen en sus rosas y haziendas todo el año y sin venir a la iglesia $^{8}$ (Castillero Calvo, 1967, p. 72).

Antes de esto, ya el obispo Antonio Calderón (por las mismas fechas) se había quejado de que:

los indios de la comarca que colinda con Remedios fueron sometidos y para su mejor sometimiento y catequización se les reunió en los pueblos de San Félix y Santiago de Guabalá a cargo de encomendadores españoles que les inspiraban en la fe cristiana pero que los obligaban a trabajar duramente (Carles, s. d.).

Por su parte, a finales del siglo XIX, otro indígena de Tolé denuncia ante el obispo de Panamá que "todos los asendado de Tole se ha enriquecido [sic] con el sudor de lo yndio" (Sarsaneda del Cid, 1977).

Hoy, en pleno siglo XXI, las estadísticas indican que no ha cambiado mucho la situación, aunque existen "caminos de penetración", edificios a los que llaman escuelas y estructuras -muchas de ellas vacías-, denominadas puestos de salud.

La nutrióloga Saracho Domínguez presenta los datos en resumen:

un envidiable crecimiento económico que alcanzó en 2012 el 10,7 \% del producto interior bruto (CEPAL, 2013)". Sin embargo, dicho crecimiento es acompañado por "un reparto muy inequitativo de la riqueza, que se refleja en la pobreza que afecta al país... Estas cifras se disparan entre la población indígena. La inmensa mayoría de la población indígena panameña vive en pobreza (ENV, 2008) (Saracho Domínguez, 2013a).

Más aún, añade que "las niñas y niños indígenas menores de 5 años padecen desnutrición crónica en un $62 \%$, cifra tres veces superior a la del panorama nacional (ENV, 2008)" (Saracho Domínguez, 2013a). Yendo más allá, se puede decir que "el actual movimiento migratorio pendular hacia zonas de agro-exportación o hacia ciudades grandes de Panamá, erosiona y afecta la subsistencia y soberanía alimentaria de estos pueblos" (Sánchez Saavedra, 2013, p. 10).

Y, hablando directamente del tema alimentario, quienes devengan el salario mínimo gastan el $75 \%$ de éste en la canasta básica, lo cual se torna más que angustioso, sobre todo en el caso de los indígenas.

8 Desde Ntra. Sra. de Los Remedios, 1 agosto de 1604, adjunto a la carta del Obispo, en AGI: Panamá, 45. La negrilla es mía.

9 Carta de Basilio Ruis Zurdo, 1 de septiembre de 1893, en Archivos del Arzobispado de Panamá (A.A.P.), Legajo C-8. 


\section{Nuestros abuelos nos enseñaron...}

¿Cuál es la razón profunda de todo esto? ¿Es solamente el efecto de la imposición colonial? ¿No hay esperanza? ¿Es el pueblo ngäbe un pueblo condenado a la extinción? ¿De dónde surge la capacidad de mantenerse en el tiempo, luchando, resistiendo, permaneciendo?

Hace años, llegué a un rancho de la comarca y había un grupo familiar (unas diez personas, entre adultos y niños) sentado alrededor del fogón donde se encontraba una paila (olla) grande, tapada, en la cual cocinaban "algo". Estuve un rato platicando y me despedí. Cuando estaba fuera del rancho me preguntaron si comía "hojas" (mä ka mrörea?). Contesté que sí y me invitaron a pasar nuevamente y me brindaron una totuma ${ }^{10}$ con una sopa de frijoles y muchas, muchas "hojas" de diferente tipo.

Esta es una de las muchas comidas heredadas de los abuelos que ha permitido mantener un poco fuera de la desnutrición a miles de indígenas. Las hojas ${ }^{11}$ son el gran "socorro" de la comida indígena: hojas de otoe ${ }^{12}$, fuente muy buena de vitaminas y minerales; hojas de uyama ${ }^{13}$, también fuente de betacaroteno y vitaminas, además de ser medicinales; hojas de frijol $^{14}$ y el mismo frijol tierno, con toda su riqueza de proteínas, vitaminas y ácido fólico (contra la anemia); berro de agua ${ }^{15}$, del cual se comen las hojas y los brotes tiernos, con toda su riqueza de iodo, hierro y vitamina A. Incluso flores y cogollos como las de la chichica ${ }^{16}$ o la flor de la cabuya ${ }^{17}$ (bon kwetdare), también son alimenticias. En fin, las "ka" o jirakas constituyen una gran fuente de nutrientes, medicinas e incluso son usadas en rituales ${ }^{18}$.

Hay alimentos -que consumen los ngäbe- muy ricos en nutrientes y poco conocidos por los no indígenas, por ejemplo el llamado "ñürün" o "bodá"19 que es un alimento prehispánico, más rico en calcio y fósforo que cualquier hortaliza. Así como los muy conocidos frijoles de palo o guand $\mathfrak{u}^{20}$, que son una gran fuente de

10 Totuma (siä en ngäbere; guacal y morro en Centroamérica y México) es una especie de recipiente para comida o bebida (o para otros usos), hecho de la mitad del fruto seco del árbol de jícaro (siägrie en ngäbere/Crescentia cujete). Es uno de los múltiples usos de este árbol.

11 Cuando hablamos de hojas, nos estamos refiriendo a vegetales verdes que, a través de siglos, se han reconocido como ricos en nutrientes. Muchos no indígenas, desde su ignorancia, califican de "cochinos" a los indígenas por consumir este tipo de vegetales. Una más de las tantas discriminaciones.

12 Otoe - tä (= Colocasia esculenta/malanga, taro, quiquisque, tiquisque), planta probablemente originaria de la India. Se come el tubérculo y las hojas tiernas.

13 Uyama - be (= Cucurbita máxima/zapallo, auyama, ayote), planta originaria de América. Se come la flor, el fruto, las hojas y las semillas.

14 Frijol - muma (= Phaseolus vulgaris), planta originaria de América. Se come el frijol y las hojas tiernas.

15 Berro de agua (= Nasturtium officinale/berro, mastuerzo), planta originaria de Europa-Asia.

16 Chichica- müne (= Heliconia latispatha), planta originaria de América. Se come la flor y el cogollo.

17 Cabuya - bon kwetdare (=Furcraea cabuya/maguey, áloe americana), planta originaria de América. Se come la flor, además de otros servicios que da. Bon kwetdare (cabuya para comer) se diferencia del bon krare (cabuya para bolsas).

18 Ver listado de once clases diferentes que podemos leer en Saracho Domínguez (2011, p. 48). De hecho, en ngäbere hoja se dice "gä" o "ka", por eso el término genérico de ka. No confundir con cantos $(=\mathrm{ka})$.

19 Ñ̈rün (= Chamaedorea tepejilote/pacaya, maíz de montaña), originario de América. Se come la flor y el palmito. Alimento muy apreciado por la población indígena maya.

20 Frijol de palo - muma krire (= Cajanus cajan / guandú, guandul, chícharo), planta originaria de África o India. 
Encuentro No. 100, 47-59, 2014

minerales (fósforo, manganeso, magnesio, ácido fólico y flavonoides). Igual se puede hablar de la fuente increíble de nutrientes que contiene el pifá ${ }^{21}$ o daba, en ngäbere, del cual se come el fruto y el cogollo o palmito, aparte de otros múltiples usos que tiene (consultar al respecto: Chízmar et al., 2009; Gupta, 2004; Miranda Allen, 2012; Montoya-Greenheck \& Campregher, 2012; Sippel \& Sanjur, 1995).

El pifá o chontaduro o pejibaye no solo es un componente esencial de varias culturas, sino que además posee bondades enormes, pues aporta proteínas, aceites, vitaminas liposolubles y minerales; en otras palabras, es como una pequeña fábrica nutricional y probablemente el alimento más completo del trópico. Un conocedor profundo de este alimento dijo que era "un cultivo de gran interés a nivel mundial para el combate del hambre, su valor nutritivo es excelente, es mejor que el maíz y prácticamente superior a cualquiera de los cultivos que consumimos como fuente de energía” (Urbina Vargas, 2008: 87).

Los ngäbe también elaboran conservas como el gwa münün ("polvo de pescado") o el kwi münün 22 ("polvo de gallina”), que tienen muchísimos nutrientes y también son desconocidas por los no indígenas de la ciudad. Además preparan el mren kugwän ${ }^{23}$ ("sal quemada"), muy rica en calcio. De igual forma se puede hablar del "entierro" del pifá para su mejor conservación (Saracho Domínguez, 2013b).

Todo esto, sin mencionar los hongos, muy apetecidos por los ngäbe, los diversos tubérculos (ñame, ñampí, yuca, otoe) y las diferentes frutas sumamente nutritivas y también medicinales, como marañón, aguacate, guayaba y papaya ${ }^{24}$, por solo mencionar cuatro originarias del continente. Al mismo tiempo, los animales de caza y de pesca que, aunque no son abundantes, también proveen de proteínas y alimento. Por supuesto, tampoco hemos hablado de la variedad de comidas tradicionales entre los guna (dule masi y moe $\left.{ }^{25}\right)$, los wounaan $\left(k a o^{26}\right)$ y los embera (bodochi y borojó ${ }^{27}$ ). Con toda esta riqueza alimenticia, la pregunta es: ¿por qué estamos como estamos?, ¿por qué es tan grave la desnutrición y la inseguridad alimentaria?

¿De dónde surge una situación tan escandalosa? De siglos de inepcias, racismo, discriminación, prepotencia, intolerancia, falta de voluntad política para hacer algo a favor del pueblo... En Panamá, y probablemente en muchos países del continente,

21 Pifá - daba (= Bactris gasipaes / pejibaye, pixvae, chontaduro, manaco), planta originaria de América. Alimento muy apreciado por indigenas de Suramérica.

22 Igual podría hacerse esta conserva de cáscara de huevo de pavo que es originario de América.

23 Mren kugwän = Se refiere a la sal recogida en la montaña ("sal negra") y cocinada con cáscaras de huevo molidas, de ahí la riqueza de calcio. La proporción cáscara-sal es de 95\%-5\%, aunque la proporción puede variar.

24 Marañón (= blü en ngäbere / Anacardium occidentale); aguacate (= duga en ngäbere / Persea americana); guayaba (= ngima en ngäbere / Psidium guajava); papaya (=kengema en ngäbere / Carica papaya). Todos ellos ricos en vitaminas, minerales y con propiedades medicinales abundantes.

25 Dule masi es la comida tradicional, que se compone de plátano, yuca, otoe... cocinados; el pescado se cocina aparte en agua de coco. El plátano se sirve con el líquido en el que se ha cocinado el pescado. El pescado se sirve aparte en seco. El moe es también otra comida guna que se prepara con uyama, coco y pescado. Es delicioso. No se puede olvidar la gastronomía del pescado asado, seco y el consumo de centollos y langostas.

$26 K a o=$ Sopa de plátano rayado, con pollo. Con la riqueza de potasio y iodo que tiene el plátano.

27 Bodochi es el bollo de maíz y borojó es una planta rica en proteínas, fósforo y vitaminas, además de ser medicinal. Tampoco se debe olvida la variedad de productos que cultivan: maíz, plátano, arroz, yuca, guineo, ñame, otoe, frutales, ñampi, frijol, uyama, caña, coco, dachin... 
se puede señalar que la causa de este tipo de situaciones está en un sistema "en el que el provecho se considera como el motor esencial del progreso económico, la concurrencia como ley suprema de la economía, la propiedad privada de los medios de producción como un derecho absoluto"(Pablo VI, 1967, N. ํ26).

Este sistema se manifiesta en políticas erradas, desviadas, contrarias a la identidad indígena, que se muestran en varios aspectos. En la agricultura las políticas han sido orientadas cada vez más hacia la agroexportación (cereales, carnes y productos no tradicionales) y hacia la agroindustrialización (azúcar, café y etanol). En la comarca ngäbe-buglé en ningún momento se han dirigido políticas agrícolas hacia la producción propia, tradicional, adaptada al ambiente, conocida por este grupo y con una gran riqueza nutricional, como se ha visto.

Los pocos proyectos agrícolas dirigidos a la comarca han sido para el cultivo de hortalizas no producidas por el pueblo indígena (cebolla, zanahoria, lechuga, repollo, entre otros), o bien para la siembra de árboles extraños (pinos, teca, eucalipto). Ciertamente, la mayor parte de los terrenos comarcales son de vocación forestal ${ }^{28}$, pero ¿por qué no se han sembrado árboles propios, frutales, sobre todo el pifá (pejibaye)? Eso sí, se ha promovido bastante la siembra de café -a veces orgánico- para engrosar el rubro de exportación. En definitiva, se trata de políticas erradas de agricultura que no mejoran la nutrición de las personas (Selles, 1998; Jaramillo Santander, 1998).

Las políticas de salud de los gobiernos han sido orientadas siempre a la medicina curativa y no a la preventiva. En las ciudades existen campañas de prevención (dengue, vacunación de niños, entre otros), pero en las zonas indígenas la cobertura se torna mucho más limitada.

En el caso de la comarca ngäbe (a diferencia de la comarca embera-wounaan), los krägä bianga y las ngibiaga ${ }^{29}$ no forman parte integral del sistema de salud; son requeridos - a lo sumo- como traductores. Toda su sabiduría preventiva y curativa no ocupa ningún espacio en el sistema oficial. Se intenta controlar las enfermedades con medicina química artificial, cuando hay muchas posibilidades de curación y prevención con las plantas medicinales, en otras palabras, políticas erradas en salud que no recuperan lo valioso del patrimonio cultural del pueblo (ver: Marco \& Sánchez, 2013).

En lo concerniente a la educación, en la escuela oficial se enseñan contenidos para posibilitar a los niños y jóvenes a ser ciudadanos y acceder a niveles de educación superior. Con estos dos objetivos -generales, aunque algo confusos y fuera de la realidad- se tienen escuelas en las zonas indígenas en las cuales (por ejemplo, en la comarca ngäbe-buglé) hay un ataque permanente a la identidad del indígena como pueblo.

En los contenidos de enseñanza apenas si se nombra la historia y el desarrollo de las culturas indígenas. Por supuesto, no se conoce, más aún, existe un verdadero menosprecio y rechazo por los idiomas, las costumbres, los ritos y la riqueza

28 Se alude aquí a la clasificación de tierras del I al VIII, según la cual la tierra tipo I es la mejor tierra (arable) y la tipo VIII es tierra mineralizada. Las tierras de la Comarca Ngäbe-Buglé están -en su mayoría- entre las tierras tipo V y VIII (Jaramillo Santander, 1998).

29 Krägä bianga (el que da medicina) $=$ médico tradicional y ngibiaga $($ la que espera $)=$ partera, ambos en idioma ngäbere. 
Encuentro No. 100, 47-59, 2014

alimentaria de esos pueblos ${ }^{30}$. En fin, se crea un bombardeo contra la identidad étnica con la consiguiente baja en la autoestima de esos pueblos. Si en las escuelas de la zona indígena no se recupera esta riqueza, se seguirá atentando no solo contra la seguridad alimentaria sino, sobre todo, contra la soberanía alimentaria de este pueblo.

Aunque, desde 1975, en Panamá se habla de educación bilingüe -al principioy de educación bilingüe intercultural, en el 2012 ha surgido el primer grupo de maestros bilingües para la comarca ngäbe. Además, solo existen algunos materiales elaborados, pero en la práctica ni siquiera se ha desarrollado la EBI (o EIB) (Bastida, 1999; Jaén, 2011; Roquebert León, 2012 y Sarsaneda del Cid, 2013).

Dados los siglos de marginación, racismo y desprecio hacia los indígenas, se ha llegado a situaciones absurdas -aunque comprensibles-, como el hecho de que los padres de familia ngäbe no les hablen a sus hijos en su idioma natal "para que no sufran lo que ellos sufrieron”, o bien, aunque existen leyes que amparan, promueven e impulsan los idiomas indígenas ${ }^{31}$, éstos con dificultad son conocidos más que por los hablantes.

En conclusión,

la producción insuficiente de alimentos, el rechazo de los modos ancestrales de producción, la ausencia de transferencias de nuevas tecnologías adecuadas y sostenibles para la realidad rural de la Comarca o para los que viven en zonas de montaña, el deterioro de los recursos naturales, un entorno socio-cultural que no potencia la capacidad adquisitiva de la población y el desconocimiento casi total -culpable o no- de los aportes culturales alimentarios del pueblo ngäbe, unido a una educación formal de espaldas a la cultura ngäbe y a una salud meramente curativa -ni siquiera universalque no integra la riqueza cultural indígena, además de la permanente 'inconsulta' a las comunidades interesadas, todos estos son factores que propician la inseguridad alimentaria de la Comarca (Idiáquez \& Sarsaneda, 2012, p. 70).

Todo lo anterior, unido a la falta de voluntad política de los gobiernos para enfrentar el tema indígena, el tema alimentario, la diversificación de la producción, la protección a los precios en el agro, entre otros, lleva al desastre humano que se vive actualmente.

\section{4. ¿Seguridad, inseguridad y soberanía alimentaria?}

Antes de tratar de buscar caminos de solución, resulta importante aclarar dos términos muy en boga actualmente y que juegan un papel vital en el tema en cuestión. En los últimos años, se ha hablado mucho de seguridad alimentaria a tal

30 Una investigadora nos compartió el haber escuchado a maestros y pastores evangélicos hablar sobre la "cochinada" que es comer las jirakas.

31 Además de las leyes comarcales que tienen alguna protección hacia los idiomas y la cultura en general, también existen otras leyes específicas como la 88 de 2010 (sobre desarrollo de idiomas y la EBI) y los artículos de la Constitución Nacional que amparan este tema (Arts. 88, 90 y 108). 
punto que se ha convertido en un término incluido y asumido en las agendas y discursos de la ONU (FAO), de las agencias internacionales de distintos "pelajes" y de muchos gobiernos y políticos de todas las fracciones.

Según la FAO, para obtener seguridad alimentaria se requiere que hayan suficientes cantidades de alimentos, y de una calidad adecuada para ser suministrados a través de una producción nacional o de importaciones (comprendida aquí la ayuda alimentaria); que se tengan los recursos adecuados para adquirir los alimentos apropiados y acceder a una alimentación nutritiva; que haya una utilización biológica de los alimentos, proponiendo como tal una alimentación adecuada, agua potable, sanidad y atención médica, para lograr un estado de bienestar nutricional; que existan insumos no alimenticios que, aunque están totalmente relacionados con la salud nutricional de una población, no se alcanzan simplemente asegurando el alimento (Saracho Domínguez, 2011, p. 66ss).

Al respecto, Saracho Domínguez (2011) comenta que "dirigir parte de los recursos económicos del Estado a la compra de productos de importación en vez de invertirlo en las causas de la mala situación alimentaria de su pueblo, ni es una solución, ni una mejora” (p. 72). Y añade:

Pareciera que el objetivo estatal fuera transformar al campesinado en una fuerza de trabajo asalariada (idónea para la industria o el sector servicios) o simplemente avocar inmensos territorios al monocultivo...

Por un lado, la Seguridad Alimentaria deja de lado la noción de campesinado y los derechos sobre la tierra y la producción de los mismos. Está demasiado inserta en el sistema económico y el comercio internacional, olvidando la potencialidad de los pequeños productores y los mercados locales... y la cultura alimentaria de la población (Saracho Domínguez, 2011, pp. 72-73).

Por otra parte, el objetivo del concepto de soberanía alimentaria, impulsado por organizaciones en por lo menos 69 países,

es mejorar la situación alimentaria priorizando los derechos fundamentales de la población campesina, pues son ellos quienes cultivan y cuidan la tierra. Este planteamiento prioriza el derecho de las poblaciones campesinas tanto al acceso a la tierra como a la producción de éstas.

Prioriza el derecho de los pueblos a definir políticas agropecuarias y de producción de alimentos. Incluye el abastecimiento de productos alimentarios a los pueblos que los producen. Plantea como objetivo primordial de la producción, cubrir las necesidades de comunidades locales. Este es un punto importante teniendo en cuenta que gran parte de la producción alimentaria de los países "en desarrollo" se destina al consumo de los países "desarrollados". Propone como necesario el derecho a proteger y regular la producción agrícola y ganadera así como el mercado nacional (Saracho Domínguez, 2011, pp. 72-73).

El potencial de su acción se fundamenta en los siguientes seis pilares: (1) Enfoca en alimento para el pueblo; (2) Valora a quienes proveen alimento; 
Encuentro No. 100, 47-59, 2014

(3) Localiza sistemas de alimentación; (4) Empodera localmente; (5)

Desarrolla conocimiento y destreza; y (6) Trabaja con la naturaleza (Saracho

Domínguez, 2011, pp. 67-68).

Saracho Domínguez (2011) concluye que la diferencia entre ambas posiciones reside en la voluntad de cambiar el sistema alimentario, y no solo la alimentación. Una vez aclaradas estas posiciones y antes de analizar cuáles caminos se podrían seguir, me gustaría proponer una corriente de pensamiento y de prácticas impulsada por organizaciones indígenas de América que enriquece lo propuesto en el presente artículo.

\section{Por el camino (¿utópico?) del Buen-Vivir (Nüne kwin gwaire)}

En América Latina se habla mucho de desarrollo económico para calificar los proyectos que se llevan a cabo y se promueven en las zonas indígenas, y la comarca ngäbe-buglé no escapa de esto. Son millones de dólares los que se han invertido en los últimos cuarenta años en esa zona y no se nota realmente el cambio a juzgar por el sufrimiento actual. Sí se evidencia en las carreteras, en edificios, en más casas, en los postes de electricidad que tímidamente van subiendo a la montaña, pero ¿y las estadísticas que no mienten?, ¿y el sufrimiento real de la gente?

En muchos pueblos originarios se está hablando tanto del "buen vivir" como de un estilo de vida (un "modo de proceder", dirían los guaraní) que implica mucho más que el simple desarrollo económico (ver: Huanacuni Mamani, 2010; Gudynas, 2011; Choquehuanca, 2010). Al respecto, los guaraní hablan de "relaciones de reciprocidad y el poder compartir” (Albó, 2009, p. 35, ver también Albó, 2003), mientras los queshwaymara lo definen en la Constitución boliviana de la siguiente manera:

La economía plural articula las diferentes formas de organización económica sobre los principios de complementariedad, reciprocidad, solidaridad, redistribución, igualdad, seguridad jurídica, sustentabilidad, equilibrio, justicia y transparencia. La economía social y comunitaria complementará el interés individual con el vivir bien colectivo (Art. 306) (Albó, 2009, p. 39).

De la misma forma, entre el pueblo ngäbe se podría hablar un poco de juritde $^{32}$ y de etdebali ${ }^{33}$, como instituciones culturales centrales en la cultura, que pueden recoger algunos aspectos del ahora llamado "vivir bien". Estos conceptos son los que nos brindan el marco cultural para plantear los posibles caminos para salir

32 Juritde es una palabra del idioma ngäbere, que no tiene traducción exacta, podría ser compartir, pedir, distribuir, ser solidarios, redistribuir. Se trata de ayudar a quienes, por alguna razón, no han tenido buena cosecha o están en situación precaria (viudas, huérfanos, enfermos).

33 Etdebali, palabra ngäbere que viene de etdeba (=hermano) y que designa la hermandad ritual adquirida a partir del krün gitde (=balsería) que es un juego ritual que acerca a las comunidades y que probablemente, en sus inicios, era una forma de redistribución y nivelación de excedentes. 
adelante con algo tan vital como es la soberanía alimentaria del pueblo (Young, 1993 y Quintero Sánchez, 1998).

\section{Por los difíciles caminos de la organización}

¿Por qué caminos transitar para revertir un proceso de siglos? ¿Cuáles mecanismos concretos se deberán abordar para que un caminar hacia la destrucción se pueda reorientar? ¿Qué elementos culturales se deben recuperar para que algunos procesos vitales como la nutrición autóctona, la ecología propia, la medicina tradicional, entre otros, puedan ser nuevamente una parte integral de esta sociedad indígena?

Lo primero, y con condición sine qua non, debe ser construir estrategias "desde abajo", es decir, desde las comunidades locales, desde las organizaciones propias, tal como lo implica el movimiento prosoberanía alimentaria. Cualquier política regional, estatal, oficial o particular que no cumpla dicha condición no dará resultados.

El pueblo ngäbe es un pueblo de consensos, no de supuestas "votaciones democráticas" y mucho menos de aceptar directrices impuestas por "autoridades" (más bien se les debería llamar poderes) de cualquier tipo. La otra condición importante es que haya verdadera voluntad política de parte de los gobiernos y de las agencias de desarrollo para empezar el camino desde aquí, desde abajo.

Una de las principales políticas que debería fomentarse es el apoyo a la producción masiva de alimentos tradicionales comprobadamente nutritivos (como los mencionados en este artículo). Esto daría una base importante, una "despensa", un respaldo, para que la gente se pueda dedicar a la siembra de árboles tradicionales (fuente de comida, materiales y medicinas) y a la organización de proyectos comunitarios de producción orgánica. Igualmente, otra política podría ser apoyar y organizar la exportación de productos cultivados de manera orgánica, y apetecidos por los extranjeros (café), o que tienen un gran potencial de nutrición (aguacates, pifá, guayabas, membrillo, marañones, ñürün, entre otros).

Como una forma de fortalecer la misma zona de la Comarca, se podría apoyar y difundir la institución cultural del juritde y el trueque de productos, basados en la otra institución cultural llamada etdebali (ver notas arriba).

Como se dijo anteriormente, habría que incluir de manera formal e integral a los médicos tradicionales y parteras en el sistema sanitario de la comarca. De esta manera, se trabajaría con respeto a la cultura, con prevención, con mayor conocimiento de la situación, con mayor cercanía a la gente; en definitiva, desde la cultura, pero con el apoyo de la medicina occidental. Así, todos los elementos nutricionales que existen en la flora propia y en las costumbres preventivas que incluyen el boin y el $k \ddot{a}^{34}$, serían parte integral de la atención en salud (Sarsaneda del Cid, 2012a; ver también 2012b).

34 Boin o boine, palabra ngäbe que se puede traducir por ayuno o prevención, limitación, prohibición, que indica una situación particular (embarazo, mordedura de serpiente, algunas enfermedades) en la cual hay que abstenerse de algunos alimentos o prácticas.

$K \ddot{~}(=$ cacao) es la planta (Theobroma cacao) que no solo tiene utilidad alimentaria y medicinal sino que es parte fundamental de varios rituales de la cultura ngäbe. 
Al ser la educación formal otro de los pilares de la destrucción cultural de la cual somos testigos, se debería reorientar las políticas educativas profundas, empezando por la interculturalidad a nivel nacional, pero no solo para las zonas indígenas, pues Panamá es un país formado por la interacción de varias culturas. Al respecto, se debe tomar en cuenta que se ha aceptado la "cultura" traída por los españoles y por otras culturas llegadas a este territorio hace casi dos siglos (chinos, hindúes, norteamericanos), pero casi siempre hemos rechazado a otras culturas que tienen quinientos años o siglos de estar presentes en el territorio (africanos, afrocaribeños e indígenas). Por esto, se debe empezar pensando que se es una nación cuya identidad está imbuida de todas esas culturas y, por tanto, la interculturalidad resulta fundamental en la educación del panameño.

En concreto, habría que desarrollar - con fuerza y creatividad- la educación intercultural bilingüe prioritariamente en las escuelas de las comarcas y en las zonas indígenas. Pero, ¿en qué va a ayudar a la nutrición el hablar el idioma propio? Los ancianos tienen un gran papel que desarrollar en las escuelas porque podrían enseñar los orígenes de estos alimentos, además de dar a conocer lo básico de la medicina tradicional y de las costumbres preventivas de salud. Incluso desarrollar una universidad intercultural indígena para investigar, profundizar y preparar personal en todos estos aspectos de salud y nutrición.

Existen otros aspectos importantes que podrían aportar en la mejora de la situación nutricional de los pueblos indígenas; entre ellos, por ejemplo, la construcción de carreteras, las buenas instalaciones de salud y educación, los lugares de almacenamiento agrícola, el beneficio que puede dar la electricidad a través de la energía solar, eólica o de minihidroeléctricas, entre otros.

Pero una cuestión fundamental también es asegurar los territorios actuales de los pueblos indígenas y ponerlos a salvo de grandes explotaciones mineras o macrohidroeléctricas. Ya se han presentado casos - como las hidroeléctricas de Valle Riscó y de Barro Blanco - en que inundan territorios indígenas, acaban con cultivos, dañan ecología, rompen con ecosistemas alimentarios y nutricionales, provocan desplazamientos, crean desarraigos, entre otros. Puede suceder lo mismo también con la hidroeléctrica del río Teribe (zona indígena naso) y con otras en estudio.

\section{Conclusión}

Se ha realizado una somera revisión de la situación nutricional, sobre todo del pueblo ngäbe de Panamá, de sus riquezas alimentarias y cómo eso contrasta de manera burda con la pobreza y desnutrición escandalosa en que viven. Además, se ha tratado de buscar las posibles causas de esta situación, por lo que se han hecho propuestas de políticas - públicas o particulares】 que podrían ser caminos de solución, teniendo siempre como horizonte la soberanía alimentaria de este pueblo.

Al respecto, se debe resaltar que el pueblo ngäbe ha sufrido, sufre y sigue sufriendo de manera insultante, y sin embargo, no se extingue, sigue caminando y dando ejemplo de lucha. Esa capacidad de resistencia (y sus consecuentes estrategias de sobrevivencia) que se notó al rechazar la dominación colonial, se sigue manifestando y puede ayudar para que todas las propuestas hechas en este escrito puedan ser llevadas adelante (Idiáquez \& Sarsaneda, 2012, pp. 305-316). 


\section{Referencias bibliográficas}

Albó sj, X. (2009). Suma qamaña. El buen convivir. Revista OBETS, (4), 25-40.

Albó sj, X. (2003). Cultura, Interculturalidad, Inculturación. Caracas: Fe y Alegría.

Bastida, M. (1999). La educación intercultural: El caso de Panamá. Sinéctica, (15), $1-14$.

Carles, R. D. (s.d.). Cuándo fueron fundados los pueblos y ciudades del Istmo de Panamá. s.d.: s.d.

Castillero Calvo, A. (1967). Estructuras sociales y económicas de Veragua desde sus orígenes históricos, siglos XVI y XVII. Loteria, (135), 74-94; (137), 55-86; (138), 76-86.

Chízmar, F. C. et al. (2009). Plantas de uso folclórico y tradicional en Panamá. Heredia, Costa Rica: Instituto Nacional de la Biodiversidad.

Choquehuanca, C. D. (2010). Hacia la reconstrucción del Vivir Bien. América Latina en movimiento, (452), 1-13.

Cooke, R. (1982). Los guaymíes sí tienen historia. En Comité Foro Guaymí (Ed.). El pueblo Guaymi y su futuro. (pp. 27-64). Panamá: Comité Foro Guaymí y Ceaspa.

Gudynas, E. (2011). Buen vivir: Germinando alternativas al desarrollo. América Latina en movimiento, (462), 1-20.

Gupta, M. (2004). Investigaciones farmacognósticas sobre la flora panameña. Anales de la Real Academia Nacional de Farmacia, (70), 839-883.

Huanacuni Mamani, F. (2010). Buen Vivir / Vivir Bien. Filosofía, políticas, estrategias y experiencias regionales. Lima: Coordinadora Andina de Organizaciones Indígenas CAOI.

Idiáquez, J. A. \& Sarsaneda, J. (2012). En búsqueda de esperanza. Migración ngäbe a Costa Rica y su impacto en la juventud. San Salvador: SJM /SJR.

Jaén, E. L. (2011). El caminar de la EIB en Panamá. Exposición en 23. Emangbunabri. Buenos Aires, Comarca Ngäbe-Buglé, Panamá.

Jaramillo Santander, E. (1998). Los suelos del área guaimí. Panamá: IDIAP.

Marco, C., L. \& Sánchez, S. K. (2013). Migración, embarazo y estrategias sanitarias para la atención de la mujer ngäbe. Panamá: Acun/Asmung/Unfpa/Minsa.

Mena García, C. (2003). La frontera del hambre: construyendo el espacio histórico del Darién. Mesoamérica, (45), 35-65.

Miranda Allen, P. (2012). Fogón de mis amores. Nukwä ja tare tigwe. Cerro Brujo Gourmet: Panamá.

Montoya-Greenheck, F. \& Campregher, C. (Eds.). (2012). Diversidad Biocultural en el Pacífico Sur de Costa Rica. La integración participativa de comunidades indígenas en estrategias para la conservación y el manejo de los ecosistemas de la región Golfo Dulce. Cuadernos de Antropología. Edición Especial. San José: Universidad de Costa Rica \& Instituto Austriaco para América Latina.

Programa de las Naciones Unidas para el Desarrollo (PNUD). (2010). Atlas de Desarrollo Humano y Objetivos del Milenio Panamá: PNUD.

Quintero Sánchez, B. (1998). Ni Jutda Ngóbe nunadi kóre. El pueblo Ngóbe vivirá siempre.

Tesis de Licenciatura no publicada, INAH, México, D. F.

Pablo VI. (1967). Carta Encíclica Populorum Progressio. El Vaticano. 
Encuentro No. 100, 47-59, 2014

Roquebert León, J. L. (2012). Educación Intercultural Bilingüe en la Comarca Ngäbe-Buglé, Revista Humanitates, (1), 71-74.

Sánchez Saavedra, K. E. (2013). Seguridad alimentaria y migraciones internas en Panamá. La Estrella. Recuperado el 05 de octubre de 2013, de https:// laestrella.com.pa/panama/politica/seguridad-alimentaria-migracionpanama/23505145.

Saracho Domínguez, H. (2013a). Trabajo por una soberanía alimentaria en Panamá. La Estrella. Recuperado el 06 de octubre de 2013, de http://laestrella.com.pa/ panama/politica/trabajo-soberania-alimentaria-panama/23503288

Saracho Domínguez, H. (2013b). El uso de conservas artesanales (pescado molido y cáscara de huevo) como estrategia local de seguridad alimentaria para una suplementación dietética sustentable en la Comarca Ngäbe-Bugle, Panamá: Un enfoque desde la soberanía alimentaria. Tesis de Licenciatura no publicada, Universidad Alfonso X. Madrid, España.

Saracho Domínguez, H. (2011). Alimentación tradicional ngäbe y soberanía alimentaria frente a la imposición de hábitos exógenos. Tesis de Maestría no publicada, Universitat de Lleida. Lleida, España.

Sarsaneda del Cid, J. (2013). Derribados, pero no derrotados. Ponencia sobre Educación Intercultural Bilingüe, Congreso de Educadores Católicos, mayo, Changuinola, Panamá.

Sarsaneda del Cid, J. (2012a). Nun tä sribire ni ngäbegroge. Algunas sugerencias para la atención en salud en idioma Ngäbere. Panamá: Acun, Asmung, Minsa, Unfpa.

Sarsaneda del Cid, J. (2012b). Reflexiones en torno a la cultura ngäbe. Tareas (142), 107-119.

Sarsaneda del Cid, J. (1979). Tierra para el Guaymí. La expoliación de las tierras guaymíes en Chiriqui. Panamá, CCSS.

Sarsaneda del Cid, J. (1977). La iglesia católica de Panamá y el indígena ngäbe. Introducción al estudio de un caso de evangelización. Tesis de Licenciatura no publicada, Universidad Centroamericana, San Salvador.

Selles, F. (1998). Primer Informe de la riqueza y estado de la biodiversidad de Panamá. Panamá: ANAM/PNUMA.

Sippel, A. \& Sanjur, M. (1995). Árboles de los ngöbe. Una alternativa para el futuro, San Félix: Inrenare, GTZ, PANg, Documento X.

Urbina Vargas, S. (2008). Entrevista con el Dr. Jorge Mora Urpí. Biocenosis, 21, (1-2), $86-90$.

Valdivia, C. (2011). Panamá. AA. VV., Diagnóstico sobre la situación de los derechos humanos de los pueblos indígenas de América Central, Tomo II, 404-466. Panamá: Oficina Regional Derechos Humanos-ONU.

Young, P. (1993). Etdebali: Un viaje al corazón del pueblo Ngóbe. Panamá: Acun. 
Anexo: Mapa de las comarcas indígenas en Panamá

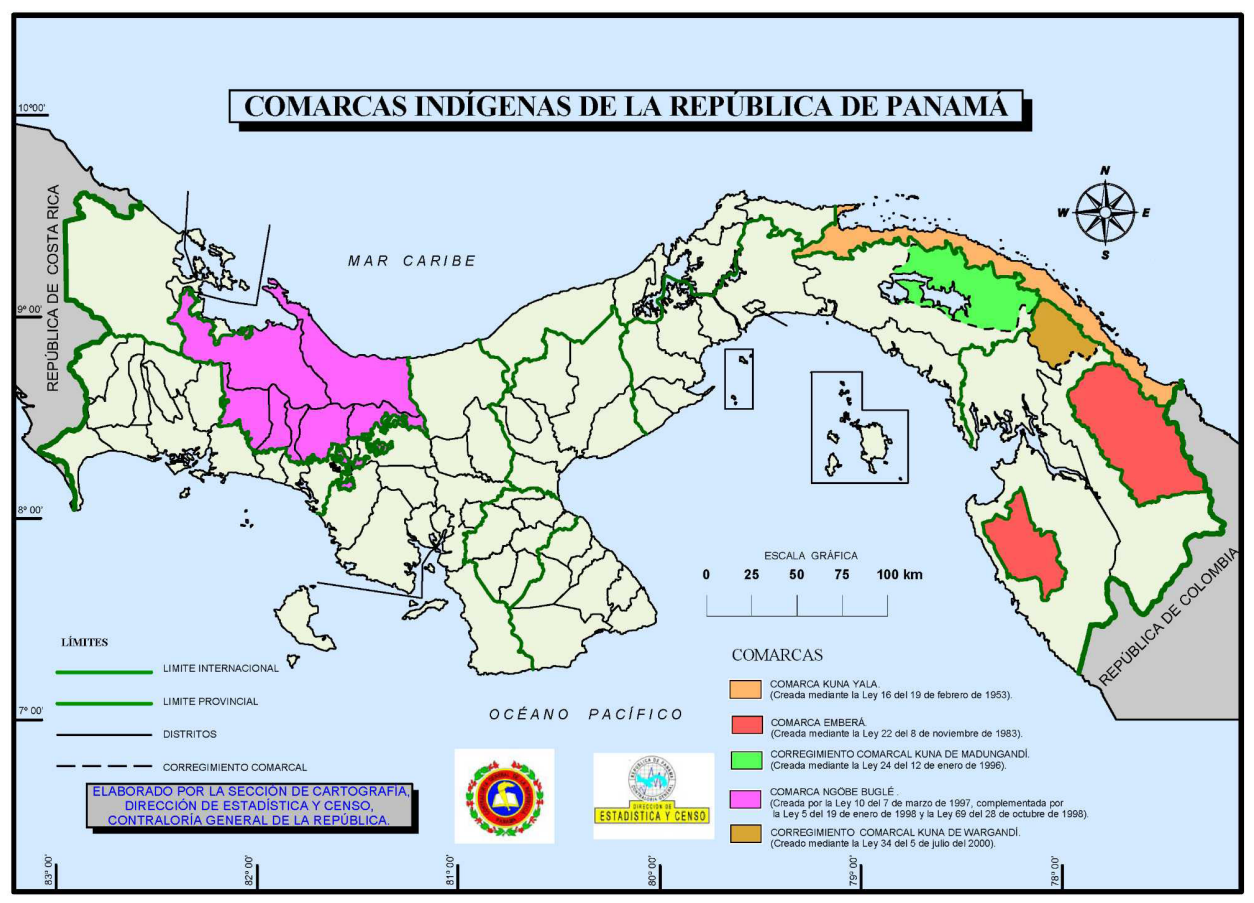




\section{Avances investigativos}

\section{Aproximación al modelo agroexportador de Nicaragua y relaciones de género}

\section{Paloma León Campos* y Johan Mosquera Góngora**}

\section{Recibido: febrero de 2015 / Aceptado: marzo de 2015}

El estudio presenta una aproximación al modelo agroexportador del banano como catalizador en la reproducción de las inequidades de género al interior de un grupo poblacional en el municipio de El Viejo, en el departamento de Chinandega, Nicaragua. La información obtenida por medio del trabajo de campo hizo posible observar las contradicciones que existen dentro del actual modelo de desarrollo agrario implementado en el país, que tiene como objetivo la producción de grandes cantidades de productos del campo destinados a la exportación. Se concluye que el modelo agroexportador en la actualidad no solo provoca un deterioro socioambiental, sino que también trae consigo una serie de problemas en la salud y malas condiciones laborales de las que se deriva la ampliación de las relaciones desiguales de género en el campo. El análisis del caso de la Clínica Xóchitl también reveló cómo, a partir de la movilización social, es posible generar propuestas de autogestión con perspectiva de género.

Palabras clave: modelo agroexportador / género / banano / Nicaragua

\section{Introducción}

América Latina es una región que cuenta con una gran biodiversidad, abundantes recursos naturales y diversidad étnica y cultural. Contradictoriamente esta región registra también altos índices de pobreza y desigualdad social.

Universidad de Østfold-Noruega, Área de Estudios del desarrollo. Correo electrónico: urpi-leon@ hotmail.com

** Área de Sociología, Instituto de Filosofía Cuba. Grupo América Latina Filosofía y Axiología Social (GALFISA). Correo electrónico: johangongora@yahoo.com 
Los informes de Naciones Unidas y la CEPAL hablan de crecimiento económico y avances en la distribución de los recursos en los últimos años (CEPAL, 2014). Pese a lo anterior, siguen existiendo expresiones de pobreza y desigualdad afincadas sobre todo en las relaciones desiguales de género. Es posible afirmar que las desigualdades de género se presentan con mayor fuerza en las zonas rurales, tributando a la ampliación de la brecha social entre el campo y la ciudad.

América Latina ha basado su producción en el uso de la tierra y la mano de obra abundante en los territorios. En éstos dominan los modelos agroproductores de la caña, el banano, los cereales y los cultivos de palma africana destinados para la exportación. El modelo agroexportador es comprendido como un modelo de desarrollo agrario que tiene como objetivo la producción de grandes cantidades de productos del campo destinados a la exportación. A pesar de que este modelo genera empleo, crecimiento económico y "uso efectivo de la tierra" de problemáticas con respecto a las relaciones desiguales entre hombres y mujeres en el ámbito laboral y de la vida cotidiana.

Este artículo busca realizar una aproximación al modelo agroexportador del banano como catalizador en la reproducción de las inequidades de género al interior de un grupo poblacional en el municipio de El Viejo en el departamento de Chinandega, Nicaragua.

Para realizar dicha aproximación tendremos en cuenta la información recopilada durante el trabajo de campo realizado en Chinandega, donde la principal fuente de información es la experiencia de la Clinica Xóchit ${ }^{2}$.

La existencia de la Clínica Xóchitl muestra cómo, a partir de la movilización social, es posible generar propuestas de autogestión con perspectiva de género. A través de esta experiencia fue posible observar las contradicciones que existen dentro del actual modelo agroexportador implementado en Nicaragua.

\section{Chinandega y la producción agroexportadora en Nicaragua}

Para entender el modelo agroexportador en Nicaragua, es importante recurrir a la historia de este país centroamericano. En los años 50 se registró en Nicaragua un incremento en la exportación de productos del campo como el algodón, el café y el banano. El gobierno de entonces recurrió a la construcción de carreteras y la creación de almacenes para productos agrícolas ${ }^{3}$.

La exportación de algodón alcanzó su pico a finales de los 70, lo que significó un crecimiento económico para el país. Sin embargo, este resultado estuvo ligado a una sobreexplotación de la fuerza de trabajo en el campo, expropiación de tierras y desplazamiento como estrategia de sobrevivecia. Con la crisis mundial de los precios

\footnotetext{
Cuando se coloca uso efectivo de la tierra bajo comillas, es porque esta afirmación genera opiniones polémicas y responde a diferentes intereses políticos y económicos.

El trabajo de campo en Chinandega fue realizado entre febrero y abril de 2014.

3 La dinastía Somoza ejerció el poder en Nicaragua desde 1934 y culminó con el triunfo de la Revolución Sandinista en 1979.
} 
Encuentro No. 100, 60-70, 2014

del algodón, Nicaragua se ve obligada a diversificar su producción, de tal modo que toma fuerza la ganadería en tierras de la región Centro-Norte del país.

Las precarias condiciones de los campesinos nicaragüenses produjeron una fuerte polarización entre el gobierno, sectores de la clase terrateniente y los campesinos. Estos últimos se organizaron en busca de mejores condiciones de vida. La situación en el campo fue uno de los elementos que produjo grandes movilizaciones hasta el triunfo de la Revolución Sandinista en 1979.

A partir de 1979, Nicaragua sufre consecuencias por la agresión política, económica y armada que acrecienta las dificultades para el desarrollo en el campo, haciendo difícil el acceso a maquinarias, insumos agropecuarios y nuevos mercados. Lo anterior estuvo acompañado de la inestabilidad de los precios de los principales productos agroexportables, como el algodón, el café y el banano (Fernández, 1994). Bajo estas circunstancias, Nicaragua enfrenta un período de inestabilidad económica y acude a la ampliación del modelo agroexportador como una salida viable para resolver la difícil situación económica del país.

Entre 1979 y 1981 la agricultura en Nicaragua representaba alrededor del 80\% de las exportaciones, ubicándose como productos importantes: el café, el algodón, la carne de res y el azúcar. Según Brizio Biondi-Morra (1990), el gobierno toma como alternativa para la producción de divisas, el aumento de la exportación de productos agrícolas; "atendiendo a la diversificación de los productos y de los mercados como medio de reducir los riesgos de la comercialización” (Biondi-Morra, 1990 p.69).

A pesar de que el el gobierno Sandinista generó grandes reformas sociales que aliviaron algunas de las condiciones de vida de la población nicaragüense, no logró introducir cambios sustanciales en su matriz productiva y siguió basando su modelo económico en clave de agroexportación (Fernández, 1994).

Históricamente América Latina ha estado anclada a la producción de materias primas para la exportación, con sus variables de acuerdo a cada país. Esto significa que aún se mantienen las dependencias para la definición de una política agraria propia. Nicaragua no es la excepción y mantiene como rubro importante para el crecimiento de su economía la exportación de lo que se produce en el campo. En este sentido, es difícil hablar de una producción para el autoabastecimiento, lo que inevitablemente genera traumas para alcanzar una plena soberanía alimentaria en el país centroamericano. El sociólogo Cirilo Otero señala que actualmente Nicaragua no se encuentra apta para enfrentar la explosión de la demanda alimentaria de su creciente población. Esto es debido a los bajos niveles de rendimiento de sus suelos y al inadecuado manejo en el uso de la tierra. La utilización de agroquímicos y pesticidas ha contribuido a la degradación del suelo (Álvarez Hidalgo, 2010). Según este experto, se necesita introducir nuevas formas de producción que logren generar sostenibilidad del suelo y del medio ambiente.

Pese a lo anterior, Nicaragua ha entrado de lleno en el negocio de la agroexportación, donde la producción de banano se ubica como una de las principales actividades para el crecimiento ${ }^{4}$ (Zúñiga Gonzales, 2010).

4 Chinandega es conocida por ser una región en donde los rubros de agroexportación como la caña de azúcar, el maní, el banano, el ajonjolí y en pequeña escala el café y la soya predominan. 
Cuadro 1. Datos de Centro de Trámites de las Exportaciones (CETREX) ${ }^{5}$ - por Volum

\begin{tabular}{|l|r|r|r|r|}
\hline Producto & \multicolumn{1}{|c|}{2000} & \multicolumn{1}{c|}{2005} & \multicolumn{1}{c|}{2010} & \multicolumn{1}{c|}{2014} \\
\hline Azúcar de caña & $191,891.52$ & $305,680.66$ & $283,253.12$ & $428,926.68$ \\
\hline Bananos frescos & $47,978.55$ & $54,858.18$ & $20,029.68$ & $22,911.52$ \\
\hline Maní & $45,155.53$ & $66,607.33$ & $66,924.22$ & $103,578.58$ \\
\hline Café oro & $81,685.38$ & $56,283.47$ & $101,959.15$ & $111,908.26$ \\
\hline
\end{tabular}

\section{Agroexportación y la producción de banano}

La producción de banano en Nicaragua emergió en los años 60 y se ubicó en territorios donde predominaba la producción de algodón, situada en el Noroccidente del departamento de Chinandega, captando la mano de obra sobrante de la producción de algodón y de la caña de azúcar.

Antes de 1979 el control de la mano de obra fue absoluto y las garantías de salud y educación eran prácticamente inexistentes para el grueso de la población. Con la Revolución Sandinista se intentó poner freno a la explotación indiscriminada de los campesinos, pero este objetivo enfrentó varios obstáculos. Las compañía trasnacional que dominaba el mercado del banano en Nicaragua, Standard Fruit Company, rompió el contrato unilateral con el gobierno Sandinista en 1981 y dejó de operar en el país centroamericano. El gobierno se vio obligado a buscar nuevos mercados para evitar que 3,500 trabajadores y más de 20,000 personas que dependían económicamente de la actividad bananera, perdieran su medio de vida (Equipo Envío, 1982).

Durante los años 80 muchos hombres se movilizaron para combatir en la guerra y las mujeres asumieron las labores en las fincas ${ }^{6}$. Los salarios eran mínimos y muchas de ellas estuvieron obligadas a trabajar más de 16 horas al día. Como resultado, una gran cantidad de mujeres entregó años de su vida al trabajo en las plantaciones de banano, con el riesgo de enfermarse por la utilización de pesticidas altamente venenosos y sin recibir ningún tipo de indemnización ${ }^{7}$ (Fernández, 1995).

En la actualidad la producción del banano sigue siendo uno de los rubros más importantes para la exportación en Nicaragua. Al cierre de 2014 se sumó un ingreso de 8,7 millones de dólares, es decir, 2,2 millones más que los reportados en

5 La información está disponible en: http://www.cetrex.gob.ni/website/servicios/princprodu14. html

6 En la década de los 80 se produjo en Nicaragua una guerra civil entre el nuevo ejército Sandinista y grupos disidentes armados. Esta guerra se prolongó hasta las elecciones presidenciales de 1990 en donde Violeta Chamorro fue elegida como nueva presidenta de Nicaragua.

7 Uno de los principales daños generados por la producción del banano tiene que ver con la utilización del Nemagón, pesticida de uso masivo por compañías bananeras para combatir gusanos microscópicos que dañan las raíces del banano. Este producto también ha sido empleado en la producción de algodón, azúcar y piña. Varios estudios han mostrado que este pesticida no solo contamina el aire, el suelo y el agua, sino que también ha causado graves enfermedades con efectos cancerígenos entre los trabajores. 
Encuentro No. 100, 60-70, 2014

2013 (datos disponibles en el Cetrex). Un proyecto impulsado por el Grupo Coen ${ }^{8}$ en Chinandega pretende incrementar la producción de banano para llegar a unos 8 millones de cajas al año, comparados con 2 millones de cajas registrados en el año 2013 (López, 2013). Segun el último informe de la Fundación Nicaragüense para el Desarrollo Económico y Social (FUNIDES) las proyecciones del gobierno de Nicaragua para alcanzar mayor crecimiento económico en los próximos años se encuentran enmarcadas en la continuidad del modelo agroexportador y la generación de condiciones favorables para la inversión extranjera en este sector (FUNIDES, 2014).

Chinandega ha representado unos de los departamentos con más potencial para la producción destinada a la agroexportación, cumpliendo así un papel importante para el crecimiento económico del país. Sin embargo, esta región también se encuentra profundamente marcada por la deterioración socio-ambiental, registrando una alta degradación de sus suelos, una concentración extremadamente alta de agroquímicos por habitante y la contaminación de los recursos hídricos

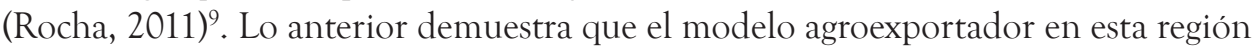
ha tenido un impacto negativo en los recursos renovables del medio ambiente, con graves consecuencias para la salud de sus trabajadores y la población en general.

De igual manera es importante resaltar las desiguales relaciones de género que se producen en la división social del trabajo en el campo, la distribución de responsabilidades y las oportunidades diferenciadas para mujeres y hombres.

El municipio del El Viejo depende directamente de las labores del campo que hoy se encuentran dirigidas hacia el fortalecimiento del modelo agroexportador. Los pobladores forman parte fundamental de la fuerza de trabajo en las plantaciones de caña, maní y banano. De las plantaciones del banano tenemos como resultado, además de las afectaciones al medioambiente, las malas condiciones de trabajo y de salud que ayudan a la ampliación de las desigualdades de género dentro un grupo poblacional en El Viejo ${ }^{10}$.

\section{Modelo agroexportador del banano y relaciones de género}

Cuando se habla de relaciones de género al interior de un grupo o proceso social, se hace referencia a las relaciones construidas entre seres humanos. Estas relaciones son construidas a través de procesos sociales y se encuentran moldeadas por tradiciones, culturas y relaciones de poder. Las relaciones de género parten de la comprensión y aceptación de prácticas diferenciadas de género, entendiendo este último como una

8 El Grupo Coen es un grupo empresarial perteneciente a una familia con poder financiero que opera en el sector agropecuario en el departamento de Chinandega.

9 Las municipalidades de El Viejo y Chinandega son las áreas en donde existe una gran concentración de producción de maní. En 2009 la producción de maní equivalía al 18\% del valor de los productos agroexportables, y solo el café $(44 \%)$ y la caña de azúcar (27\%) los superaron. El territorio de Chinandega está cubierto en un $10 \%$ por la produccion de maní. Para más información ver Rocha (2011).

10 El grupo poblacional al que se hace referencia fueron fuente principal de información en el trabajo de campo. Se trata de las personas que son impactadas de manera directa por el trabajo que realiza la Clínica Xóchitl. 
construcción social de los roles y responsabilidades de hombres, mujeres y personas con preferencias sexuales diversas. En el marco de las relaciones sociales de género, es generalmente la mujer quien tiende a ser el sujeto más vulnerable, con posibilidades de decisión y participación más limitadas. El enfoque de género se enmarca en el desarrollo de iniciativas que propician una relectura que promueva la igualdad de oportunidades entre las personas.

En los últimos años América Latina ha encendido sus alarmas por el aumento de las desigualdades de género, lo que pone en evidencia las complejidades que surgen para avanzar hacia un escenario de oportunidades en igualdad para todas las personas sin distinción alguna. La pobreza es uno de los principales factores generadores de violencia y desigualdad social de género, haciendo que las mujeres, sobre todo las del campo, sean las principales víctimas (Lamas, 2006).

En el caso de la producción de banano para la exportación, siendo una actividad con una historia compleja en el continente, encontramos que las mujeres enfrentan mayores dificultades en el trabajo y en el desarrollo de su vida cotidiana. En la primera década del presente siglo la situación de la mujer en las plantaciones de banano en Nicaragua no fue muy alentadora en materia de trabajo, salud y oportunidades para la inclusión social ${ }^{11}$.

Para entender el impacto que ha tenido el modelo agroexportador de banano sobre las relaciones de género en un grupo poblacional del municipio de El Viejo, tomaremos en cuenta dos factores importantes. Primero, las condiciones de las mujeres directamente involucradas en las plantaciones de banano y, por otra parte, la repercusión de la actividad bananera agroexportadora en la vida cotidiana de las mujeres.

\section{Relaciones diferenciadas de género en las plantaciones}

\subsection{Condiciones laborales}

a. A pesar de que hay presencia de mujeres en la producción de banano, son los hombres quienes constituyen la mayor fuerza de trabajo. Éstos dominan el espacio público/productivo mientras las mujeres están relegadas al trabajo doméstico/reproductivo. Igualmente, según las discusiones en los talleres durante el trabajo de campo "existe un trato discriminatorio por sexo y por edad que afecta a las mujeres a la hora de las contrataciones bananeras”.

b. A partir del año 2000 se han registrado cambios en el mercado internacional de banano, lo que ha significado una reducción de personal y de salarios. Estos cambios han provocado una alteración en la organización técnica del trabajo, generando un aumento en el esfuerzo físico entre trabajadoras y trabajores ${ }^{12}$.

11 Un informe de la Coordinadora Latinoamericana de Sindicatos Bananeros y Agroindustriales muestra las dificultades que enfrentan las mujeres bananeras en el campo nicaraguense. Ver: (COLSIBA, 2001).

12 Cifras anunciadas por miembros de la Federación de Trabajadores Bananeros de Chinandega en los talleres organizados por la Clínica Xóchitl el 28 de marzo de 2014. 
Encuentro No. 100, 60-70, 2014

c. Las mujeres han trabajado tradicionalmente en el empaque de cajas de la fruta para la exportación. Este trabajo consiste en seleccionar la fruta, pesarla y colocarla en las cajas, entre otros. Según testimonios, "para lograr efectivizar el trabajo en las empacadoras, las mujeres han estado bajo mayor presión para alcanzar un aumento en la producción de cajas de banano por día, lo cual ha significado un aumento en las horas laborales legalmente establecidas".

d. Por otro lado, el empaque depende de la época de corte de la fruta, lo que significa para las mujeres una inestabilidad laboral, y una mínima protección de seguridad social y de derechos laborales.

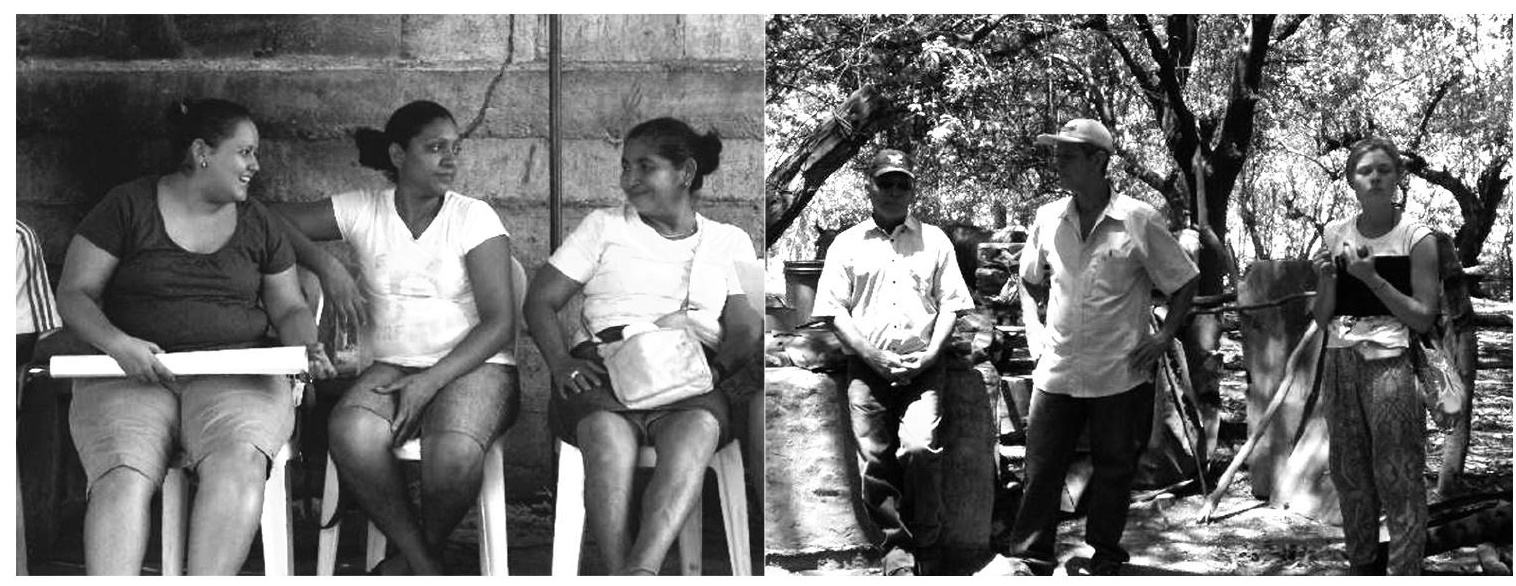

Ilustración 1. A la izquierda, taller organizado por la Clínica Xóchitl. A la derecha, trabajo de campo en finca orgánica de banano.

Fotos de Paloma León.

\section{Relaciones diferenciadas de género en la vida cotidiana}

\subsection{Condiciones de salud}

a) Las desigualdades de género tienden a ampliarse debido a las enfermedades registradas en hombres y mujeres trabajadores en las plantaciones. Por un lado, cuando los hombres se enferman las mujeres asumen el cuidado del hombre en cuanto a la enfermedad y la manutención. Esto significa un aumento en la carga de trabajo para las mujeres en el hogar. Según nuestros testimonios, "las condiciones de trabajo en las plantaciones no garantizan un seguro para solventar los gastos de manutención en el hogar en caso de enfermedad”, lo que significa que la mujer está obligada a buscar ingresos económicos por otros medios informales. 
b) Igualmente, existen algunos casos en los que las mujeres reemplazan a los hombres en las plantaciones. Sin embargo, las actividades realizadas por las mujeres en las plantaciones tienden a ser, en su gran mayoría, reconocidas con menor remuneración económica. Esto significa que el valor del trabajo es diferenciado basándose en relaciones desiguales entre hombres y mujeres.

d) El impacto de los químicos utilizados en las plantanciones bananeras también ha traído consigo problemas graves de fecundación y muchas veces abortos repentinos. Apariciones de cuadros cancerígenos que no son detectados a tiempo son problemas recurrentes entre las mujeres. Muchas de ellas han presentado enfermedades ligadas a la ocupación bananera, especialmente aquellas debidas al uso de pesticidas y las causadas por la humedad presente en las plantas empacadoras. Las enfermedades más comunes que se han registrado en la zona entre las mujeres bananeras son:

- Enfermedades nerviosas y respiratorias

- Lesiones por accidentes

- Fatiga

- Envenenamiento crónico y agudo por sustancias insecticida

- Dermatitis crónica por hongos

- Enfermedades pulmonares y alergias

- Artritis

- Hipertensión arterial

c) Dentro de las causas principales del deterioro de la salud en las mujeres encontramos las pocas garantías para el acceso a la salud, sobre todo las de tipo preventivo. Esto último ha sido el detonante de múltiples muertes entre la población femenina, entre las que se destacan las muertes por el cáncer de cuello uterino.

\subsection{Violencia en el hogar}

a) La idea de que, ante una enfermedad en el hombre, sea la mujer quien solvente el hogar económicamente y ocupe así el lugar público en una esfera de la sociedad, produce entre los hombres un deterioro de la autoestima y celos. Esto provoca confrontaciones que empeoran la situación familiar, en donde las principales víctimas son las mujeres, las niñas y los niños.

b) Esta situación se agrava para la mujer cuando ella tiene que salir a resolver el problema de la manutención, que es resuelto, en la mayoría de los casos, a través de labores informales. Según nuestros informantes, las actividades comunmente realizadas para obtener un mayor ingreso económico para el hogar son las ventas casuales y el trabajo doméstico.

c) Finalmente, a pesar de que las mujeres perciben un salario por su trabajo, éstas no deciden sobre la inversión del mismo. Esto muestra que las mujeres aún no han logrado un empoderamiento real de su condición de mujer trabajadora. 
Encuentro No. 100, 60-70, 2014

\section{La Clínica Xóchitl}

El aumento acelerado de las enfermedades ligadas a la producción bananera para la exportación, y el empeoramiento de las condiciones laborales para las mujeres, genera la creacion de la Clínica Xóchitl en 1999 como respuesta a los problemas de salud presentes en la población.

La Clínica Xóchitl es una iniciativa que nace en el municipio de El Viejo, Chinadenga, Nicaragua. Es un proyecto impulsado fundamentalmente por mujeres de la comunidad, que busca mejorar las condiciones de vida a través de la promoción del cuidado de la salud de su población. El trabajo se desarrolla a partir de la gestión de profesionales de la salud para la atención de la población, y la coordinación de seminarios y talleres formativos en temas de salud preventiva. La gestion de la clínica es realizada con el trabajo voluntario de los participantes: mujeres cabeza de familia, promotoras de salud, miembros del sindicato y personal voluntario nacional e internacional.

Actualemente la Clínica ofrece consultas ginecológicas, psicológicas, pediátricas y de medicina general. Las actividades de la Clínica tienen un impacto importante en las comunidades de Buenos Aires, el Mitch, La Unidad y Colonia San Gerónimo.

Esta experiencia también ha contribuido a establecer un díalogo sobre las formas en que la violencia de género se genera y se reproduce en las comunidades. La participación y testimonios de hombres en los talleres también han sido claves para descifrar algunas manifestaciones de violencia que están estrictamente ligadas a razonamientos machistas.

Uno de los aportes importantes de la Clínica es que ha logrado aglutinar a los actores afectados por la producción de banano para la exportación. La realización de talleres y reuniones en los que participan trabajadores y ex trabajadores de las plantaciones permiten dar un seguimiento sobre sus condiciones laborales y de salud. Esto es posible gracias al estrecho vínculo que guarda esta organización comunitaria con el sindicato bananero.

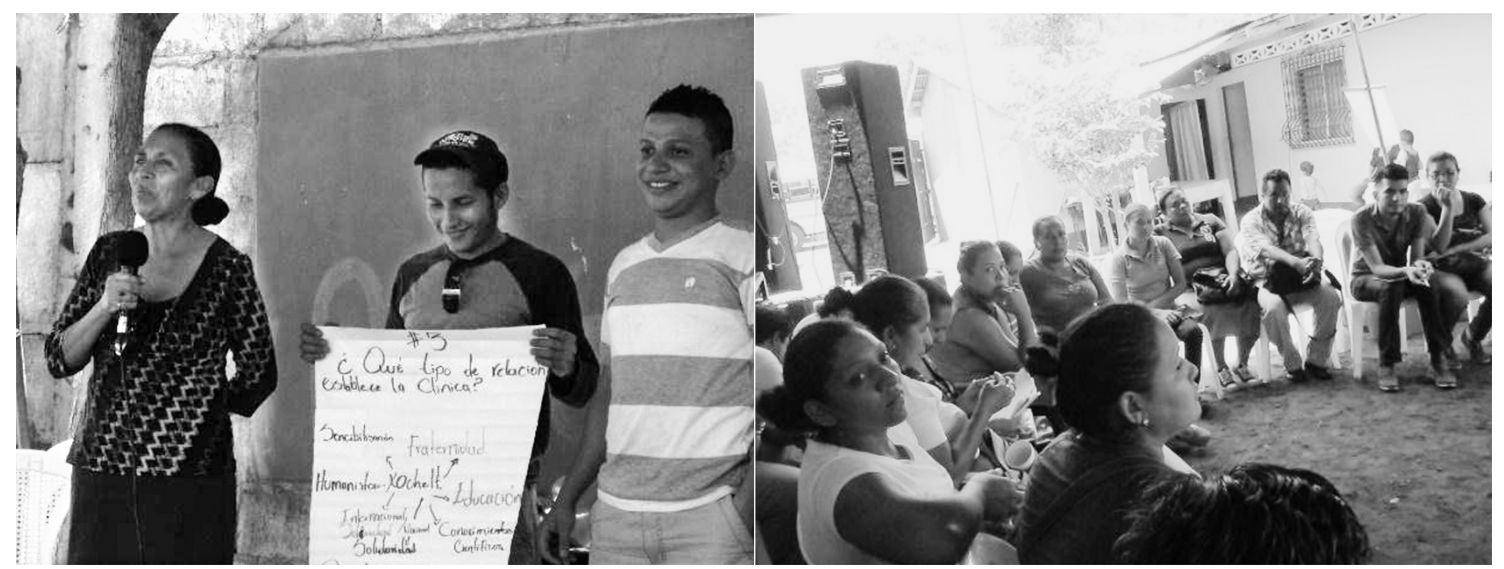

Ilustración 2. Taller formativo organizado por la Clínica Xóchitl Fotos de Paloma León. 


\section{Consideraciones finales}

La discusión sobre el modelo agroexportador y su impacto sobre las relaciones de género tiene un papel importante a la hora de definir políticas que apunten al desarrollo integral de la sociedad. Esto implica un marco de posiblidades para mejorar la calidad de vida de hombres y mujeres en igualdad de condiciones.

La agroexportación de banano en el municipo de El Viejo, Chinandega, ha tenido múltiples impactos en el ámbito laboral y en la vida cotidiana de las personas involucradas. La búsqueda de una producción efectiva y de calidad, junto con el incremento del desempleo, han hecho que el trabajo físico de las fincas aumente significativamente. El trabajo por temporada junto con grandes diferencias de salario entre hombres y mujeres hace que las mujeres sean las más vulnerables ante la inestabilidad laboral y mínimas garantías de seguridad social.

La utilización de químicos en las plantaciones ha provocado graves problemas de fecundación y abortos repentinos entre las mujeres. La aparición de cuadros cancerígenos que no son detectados a tiempo es también otro problema recurrente entre las extrabajadoras. Esto está ligado a las pocas garantías que tienen para el acceso a la salud, sobre todo, la de tipo preventivo. Esto último ha provocado múltiples muertes entre la población femenina, donde se destacan las muertes por el cáncer de cuello uterino. Por último, el modelo agroexportador del banano también ha tenido su impacto indirecto en la vida cotidiana de las mujeres, quienes han sido expuestas a ciclos de violencia en el hogar.

La experiencia de la Clínica Xóchitl, de ser potenciada, permitiría hacer frente a la difícil y compleja situación de las mujeres en las zonas bananeras. La forma en como esta organizado el modelo agroexportador en la actualidad no solo provoca un deterioro socio-ambiental, sino que también trae consigo una serie de problemas en la salud y malas condiciones laborales de las que se derivan la ampliación de las relaciones desiguales de género en el campo.

Lo expuesto invita a pensar en un modelo de producción enfocado en el respeto de los derechos de la naturaleza y de los seres humanos. Esto implica una visión integral del modelo agroexportador y sus impactos en el medio ambiente y la población.

\section{Referencias bibliográficas}

Álvarez Hidalgo, W. (2010). País necesita más productividad. La Prensa. Recuperado el 30 de enero de 2015, de http://www.laprensa.com.ni/2010/07/27/ economia/32747-pais-necesita-mas-productividad

Biondi- Morra, B. (1990). Revolución y política alimentaria: un análisis crítico de Nicaragua. México: Siglo XXI.

CEPAL. (2014). Perspectivas económicas de América Latina 2015: Educación, competencias e innovación para el desarrollo. Comisión Económica para América Latina y El Caribe. Recuperado el 15 de enero de 2015, de http://repositorio.cepal.org/ bitstream/handle/11362/37445/S1420759_es.pdf?sequence=1

COLSIBA. (2001). Diagnóstico participativo con enfoque de género sobre condiciones 
Encuentro No. 100, 60-70, 2014

sociales, económicas, laborales y organizativas de las mujeres trabajadoras bananeras. Informe de la Coordinadora Latinoamericana de Sindicatos Bananeros y Agroindustriales. Recuperado el 15 de enero de 2015, de http://www. colsiba.org/sites/default/files/archivos/DPEG_\%20INFORME_FINAL_ REGIONAL.pdf

Equipo Envío. (1982). El retiro de la bananera Standard Fruit: ¿Una agresión concientizadora? Envío, 17. Recuperado el 27 de enero de 2015, de http:// www.envio.org.ni/articulo/63

Fernandez, R. (1994). Agricultura orgánica: más racional, más amorosa. Envío,146. Recuperado el 27 de enero de 2015, de http://www.envio.org.ni/articulo/839 Fernandez, R. (1995). El Nemagón en el banquillo: acusan los bananeros. Envío, 157. Recuperado el 25 de enero de 2015, de http://www.envio.org.ni/articulo/109 FUNIDES. (2014). Informe de Coyuntura Económica: Cuarto Trimestre 2014, Nicaragua. Recuperado el 1 de febrero de 2015, de http://www.funides. com/documentos/coyuntura/2014/cuarto-trimestre/cuarto_informe_de_ coyuntura_economica_de_2014.pdf

Lamas, M. (2006). Usos, dificultades y posibilidades de la categoría género. En M. Lamas (Comp.). El género: La construcción cultural de la diferencia sexual. México: PUEG/Miguel Angel Porrúa, Colección, Mexico.

López, L. (2013). Aumentará producción de banano. El Nuevo Heraldo. Recuperado el 20 de enero de 2015, de http://www.elnuevodiario.com.ni/economia/295273

Rocha, J. L. (2011). Chinandega's Navigation In the Sea of Globalizacion. Envio, 357. Recuperado el 23 de enero de 2015, de http://www.envio.org.ni/ articulo/4329

Zúñiga Gonzales, C. A. (2010). Nuevo modelo de participación ciudadana: Identificación de sistemas de producción agropecuarios y forestales en los departamentos de León y Chinandega, 2008-2009. Universidad Nacional Autónoma de Nicaragua, León. Recuperado el 25 de enero de 2015, de http://ageconsearch.umn.edu/ bitstream/56692/2/Paper_Identificaci\%C3\%B3n\%20de\%20Sistemas\%20 de\%20Producci\%C3\%B3n\%20Agropecuarios1.pdf 